\title{
Quantitative infectivity assay for HIV-1 and -2
}

\author{
P.L. Nara and P.J. Fischinger
}

The development of a cytomorphologic infectivity microbioassay for human immunodeficiency virus (HIV) allows rapid, sensitive, and specific characterization of neutralizing antibodies or antiviral agents.

INFECTION with the human immunodeficiency virus (HIV) leads to a plethora of humoural and cellular immune responses ${ }^{1}$, which ultimately leads to AIDS in humans. The characterization and understanding of these immune responses in humans may allow for the identification of various states of protection from disease, opening up new avenues for future vaccine development and for interventive strategies such as antiviral agents and biological response modifiers. To this end, a dissection of host responses by sensitive, reproducible infectivity bioassays will be of obvious importance.

In the study of bacterial viruses, the production of plaques by single virus particles (bacteriophages) on a uniform bacterial layer was the earliest and most sensitive method for evaluating various anti-bacteriophage antisera ${ }^{2}$. Dulbecco introduced to animal virology a modification of the bacteriophage plaque assay that is now used and applied widely both for the quantitation of human and animal viruses and for neutralizing antibody ${ }^{3,4}$.

The virus neutralization/infectivity test is the most sensitive and specific serological procedure for demonstrating the quantitative relationship between virus and indicator cells. In such a test, a single infectious unit of virus infects a single cell and initiates a focal cytomorphologic expression of that infection, such as syncytium formation, plaque formation, cell transformation or lysis (Fig. 1). Because a single infectious unit leads to a single discrete response, a linear relationship exists between the number of virallyinduced cytopathic effects and the first order of virus concentration ${ }^{3.4}$. When this test is applied in assays for virus inactivation or neutralization, the complex interactions of virus and antibody can be systematically and accurately evaluated ${ }^{4}$. The present assay ${ }^{5}$ has been developed to provide a relatively rapid, quantitative, and reproducible bioassay for the detection and characterization of neutralizing antibody and other candidate antiviral agents.

\section{Syncitium-forming assay}

The assay is based on the previously reported interaction between fusigenic virus-infected cells expressing the HIV envelope gene products and uninfected adjacent cells bearing CD4 molecules". The conditions are optimized in vitro so that a virus inoculum introduced onto a syncitium-sensitive Leu 3a-positive CEM
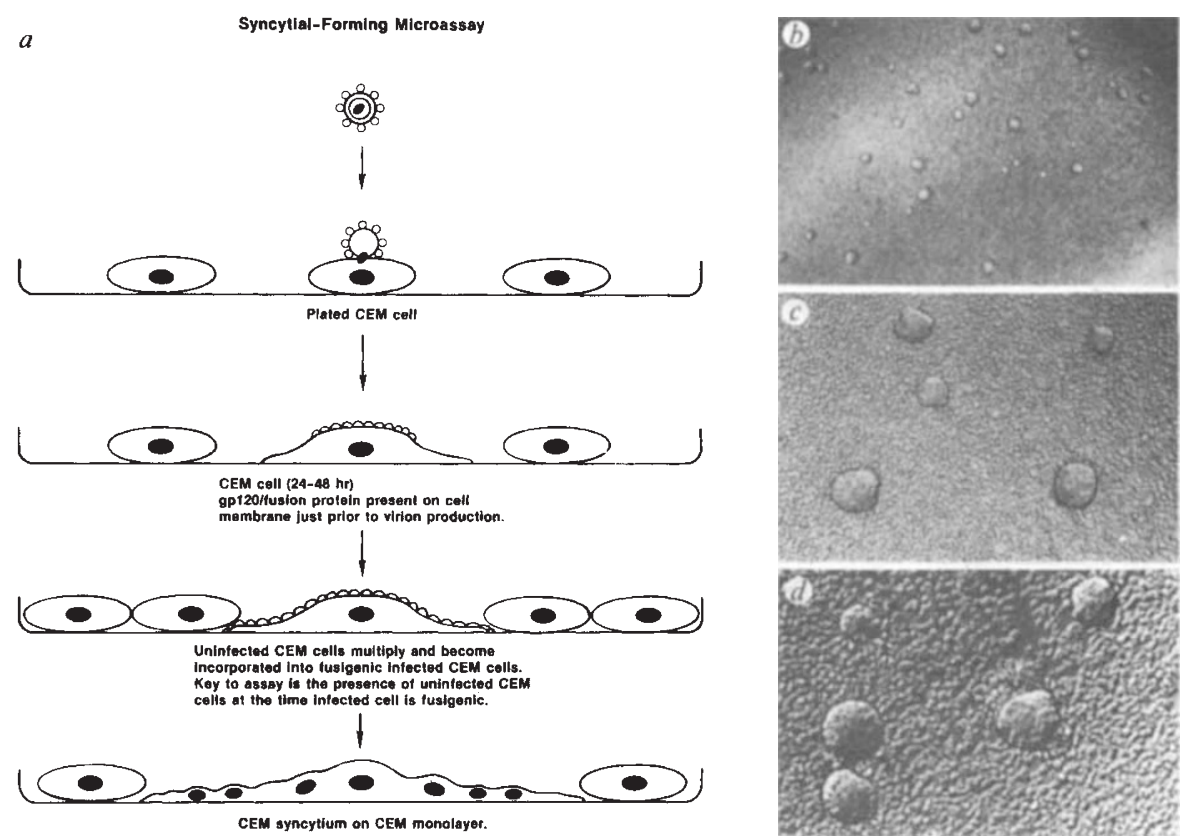

Fig. $1 a$, Schematic of the CEM-SS virus-induced syncytium forming assay (reprinted with permission ${ }^{5}$ ). $b$, Microtitre well containing 35 typical HIV virally-induced syncytia. Approximately 30 per cent of the well is shown. $c$, Five HIV-induced syncytia embedded in a monomorphic CEM-SS monolayer. Magnification, $100 \times . d$, Infectious cell centre assay. The five syncytia shown result from contact with virus-producing cells for 18 to 24 hours. Magnification, $200 \times$.

cell line (CEM-SS) monolayer leads to a population of virus-infected (fusigenic) HIV envelope-expressing cells (Fig. 1a). These cells contact the remaining pretitred population of uninfected CEM-SS cells, allowing the early recruitment of uninfected cells into a detectable and quantifiable syncytium. With this method, agarose overlays are not needed because satellite syncytia from first- and secondgeneration virus progeny are not produced within the assay period.

In the assay, individual wells of a 96-well, flat-bottomed microtitre plate (Costar) are coated with $50 \mu \mathrm{l}$ of a $50 \mu \mathrm{g}$ $\mathrm{ml}^{-1}$ poly-L-lysine solution (PLL) (Sigma) and allowed to stand at room temperature for 30 to 60 minutes, after which residual PLL is removed by two washes with physiologic buffered saline solution (PBS) or medium. During the PLL treatment of the microtitre plates, the non-adherent CEM-SS cells ${ }^{5}$ are prepared for plating. To standardize plating conditions and assure a population of logarithmically growing cells, the CEM-SS cell cultures are split in a $1: 2$ ratio 24 hours prior to plating in RPMI 1640 medium containing 10 per cent fetal calf serum, one per cent antibiotics (PSN 100X Gibco Labs) and
0.5 per cent Nystatin (optional). The cells are counted, then gently pelleted at 800 r.p.m. for five minutes. The original volume of tissue culture medium is decanted and replaced with one-tenth the volume of complete RPMI 1640 medium containing $25 \mu \mathrm{g} \mathrm{ml}^{-1}$ diethylaminoethyldextran (DEAE-D) for 30 minutes. DEAE-D may be omitted depending upon experimental conditions, but its presence assures maximum and more uniform viral kinetic infection of the cells. The cells are washed twice and resuspended in medium, and plated in the PLLpretreated microtitre plates at a final cell concentration of 50,000 cells per well in a volume of $50 \mu \mathrm{l}$. Ten ml of $1.0 \times 10^{6}$ cells per $\mathrm{ml}$ is appropriate for one 96-well microtitre plate. The cells are allowed to attach for 30 minutes at 100 per cent humidity, $37^{\circ} \mathrm{C}$ and five per cent $\mathrm{CO}_{2}$.

Frozen HTLV-III ${ }_{\mathrm{B}}$ and $\mathrm{HTLV}-\mathrm{III}_{\mathrm{RF}}$ (isolates of HIV-1) stocks are made from recently infected $\mathrm{H} 9$ cells by centrifugation. The virus-containing media is aliquoted into $1 \mathrm{ml}$ volumes and frozen at $-70{ }^{\circ} \mathrm{C}$. Viral stocks are then thawed and evaluated for syncytium forming units (SFU) by twofold endpoint dilution analysis in the CEM-SS assay. For confirma- 
tion, tests for reverse transcriptase and p24 content by radioimmunoassay can be performed simultaneously. Other HIV variants - such as HTLV-MN, HTLV$\mathrm{CC}$ and HIV $-2_{\mathrm{NIH-Z}}$ - are labile and often lose titre on freezing, and must be maintained as freshly infected cultures. Optimal tissue culture conditions have been standardized, and reproducible virus yields can be achieved on a weekly basis and be titred directly in the assays.

Frozen, pre-titred or fresh virus stocks are diluted in medium to yield approximately 100 to 200 SFU per well. Fifty $\mu \mathrm{l}$ of approximately 100 SFU of virus (the constant component) is added to $50 \mu \mathrm{l}$ each of a serially prediluted sample of antiserum, purified immunoglobulin or other potentially virus-inactivating compounds under study. These virusantibody mixtures are incubated for various times depending upon experimental conditions. Following incubation, each virus-antibody mixture is divided into duplicate wells $(50 \mu \mathrm{l}$ per well) containing PLL-adherent CEM-SS cells (in which the medium has been removed gently) for 60 minutes. Following this adsorption phase, which lasts 60 minutes for most strains, the virus-media only and virus-antibody mixtures are removed and replaced with 100 to $200 \mu \mathrm{l}$ of media. Some wells of plated CEM-SS cells are left uninfected to serve as cytomorphologic controls. The microtitre plates are stored in an incubator at 100 per cent humidity, $37^{\circ} \mathrm{C}$ and five per cent $\mathrm{CO}_{2}$ for five days. On the third day, $100-200 \mu$ l of complete medium may be added per well. On the fifth day, the plates are removed, the covers are removed, and a clear adhesive film (Plate Sealer, Linbro, Flow Labs) is placed over the microtitre wells. The covers then are replaced and each entire well can be counted for syncytia with an inverted microscope at a magnification of 40 to $100 \times$ (Fig. $1 b$ and $c)$.

Initially, the uninfected CEM-SS cells are inspected for normal cell growth and viability, as determined by confluency of the monolayer at five days. This can serve as a toxicity marker when antiviral compounds are being investigated. Next, syncytia in all duplicate wells that received virus-media only are counted. This indicates if an adequate input of syncytium forming virus was added to the assay system. The average number of syncytia obtained for the virus-media containing wells is designated $V_{o}$, for original virus inoculum. The syncytia then are counted in the duplicate wells that received the various virus-antibody mixtures. The average number obtained here for each serum dilution tested is designated $V_{n}$, for virus inoculum sur-viving after neutralization. After all of the wells have been counted for syncytia and have been averaged, the ratio of the number of syncytia induced by the virus inoculum surviving after virus neutralization $\left(V_{\mathrm{n}}\right)$ to the number of syncytia induced by the virus inoculum $\left(V_{\mathrm{o}}\right)$ is obtained. The data are represented as classical multiplicity curves $^{4}$. The virus-surviving fractions $\left(V_{n} /\right.$ $V_{\mathrm{o}}$ ) from each twofold serum dilution are then plotted on the logarithmic $\mathrm{Y}$ axis, and the reciprocal of the serum dilutions are plotted on the arithmetic $X$ axis. Each curve represents the virus-surviving fraction at progressive twofold serum dilutions (Fig. 2). Neutralization titres can be standardized at any level of per cent neutralization depending upon the individual experimental parameters. For HIV, a 90 per cent $\left(V_{\downarrow} / V_{0}=0.1\right)$ point of virus inhibition is recommended as a standard neutralization index because of the variable initial slope of the curve at high serum dilutions.

Two minor modifications to the HIV-1 assay are necessary for HIV-2 because of the more rapid viral-infectivity replicative kinetics exhibited by HIV-2 $2_{\mathrm{NIH} \cdot \mathrm{Z}}$ in this cell line. For HIV-2, 75,000 CEM-SS cells instead of 50,000 cells should be plated per well, and HIV-2-induced syncytia should be quantified on the third or fourth day instead of on the fifth or sixth day as previously described.

Several variants of simian immunodeficiency virus (SIV) have been tested with this assay to date. Several pathogenic strains of SIV/Delta have been found to replicate rapidly to maximal titres and to produce quantifiable syncytia in the CEM-SS cell line (M. Murphy-Corb, personal communication). The exact conditions of the assay are currently being defined, but the fact that some strains appear to work in the assay is encouraging. Two other strains, SIV/Mac and SIV/ Mne, have been found to replicate to a lesser extent, and do not induce quantifiable syncytia formation (P. Johnson and R. Benveniste, personal communications, respectively).

\section{Infectious cell centre assay}

The assay described above may be modified for the evaluation of infectious cell centres (ICC). Suspensions of cells that have been latently or productively infected by HIV-1 and -2 and produce virus or fusigenic proteins can be detected by this assay. The syncytia develop in 18 to 24 hours and are counted as described previously for HIV-1 (Fig. 1d). Specifically, CEM-SS cells are prepared and plated as described for HIV-1 and -2 infectivity assays, but a concentration of 100,000 cells per well is required. Cells to be quantified for virus production are washed three times in complete RPMI 1640 to remove any residual cell-free virus. The cells are then added to the previously plated adherent CEM-SS cells in tenfold incremental amounts ranging from 100 to 100,000 cells per well. In the evaluation of peripheral blood mononuclear cells from

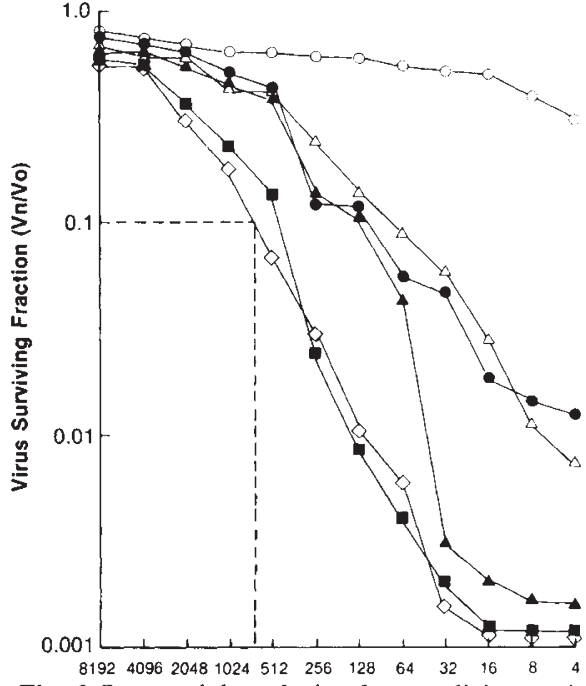

Fig. 2 Sequential analysis of neutralizing antibody response in an experimentally inoculated, persistently infected chimpanzee. The dashed line represents the 90 per cent neutralization $\left(V_{v} / V_{o}=0.1\right)$ serum titre (512-1024) from a serum sample taken 2 yr $(\diamond)$ after inoculation (AI). Prebleed (O), 1 month AI (•), 2 months

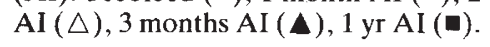

infected humans, chimpanzees, or other primates, medium containing either interleukin-2 or phytohaemagglutinin - used to support growth and viral expression do not adversely affect the CEM-SS cells.

Isolates of HIV-1 and -2 other than those reported here need to be evaluated by twofold, endpoint-limiting dilutionamplification analysis ${ }^{5}$ for their quantitative relationship to syncytia formation and the detection of non-syncytial variants. The isolates used here have been found to be free of non-syncytial variants. Should non-syncytial variants exist with other isolates, they can be evaluated in this assay system by removing supernatants and cells for viral antigen detection by radioimmunoassay, reverse transcriptase or immunofluorescence assays.

The CEM-SS virus-induced, syncytiumforming microassay should provide AIDS researchers with a rapid, quantitative, reproducible bioassay for the sensitive evaluation of multiple samples for antiviral or neutralizing activity against HIV$1,-2$, and some SIV strains.

Peter L. Nara is at the National Cancer InstituteFrederick Cancer Research Facility, Bldg 560, Room 12-15, Frederick, Maryland 21701. Peter $J$. Fischinger is at the Department of Health and Human Services, Public Health Services, 729-H Humphrey Bldg, 200 Independence Avenue, S.W., Washington, DC 20201. For more information, fill in reader service no. 100.

1. Ho, D.D., Pomerantz, R.J. \& Kaplan, J.C. New Engl. J. Med. 317, 278-286 (1987)

2. Delbrück, M. J. Bact. 50, 137-150 (1945)

Dubecco, R. Proc. natn. Acad. Sci. U.S.A.38. 747-752 (1952).

4. Dulbecco, R., Vogt, M., \& Strickland, A.G.R. Virology 2 162-205 (1956).

Nara, P.L. et al. AIDS Res. Hum. Retroviruses 3, 283-302 (1987).

6. Sodroski. J., Goh, W.C., Rosen, C., Campbell, K. \& Haseltine, W.A. Nature 322, 470 (1986). 